(C) 2013 Plant Management Network.

Accepted for publication 15 January 2013. Published 22 April 2013.

\title{
Downy Mildew from Lake Erie Vineyards is Diverse for the G143A SNP Conferring Resistance to Quinone Outside Inhibitor Fungicides
}

\author{
Christopher T. Gee, Stephanie Chestnut, Eilene Duberow, \\ Department of Biology, School of Science, Penn State Erie, The \\ Behrend College, Erie, PA 16563; Andrea Collins, Department of \\ Biology, Arizona State University, Tempe, AZ 85281; and Michael A. \\ Shields, Code Circles LLC, East Lansing, MI 48823
}

Corresponding author: Christopher T. Gee. ctg10@psu.edu

Gee, C. T., Chestnut, S., Duberow, E., Collins, A., and Shields, M. A. 2013. Downy mildew from Lake Erie vineyards is diverse for the G143A SNP conferring resistance to Quinone outside inhibitor fungicides. Online. Plant Health Progress doi:10.1094/PHP-2013-0422-01RS.

\begin{abstract}
Downy mildew (Plasmopara viticola) is a significant problem in grape vineyards throughout the growing season. Control of downy mildew is carried out with a combination of host tolerance and chemical applications. Even in vineyards planted with very tolerant varieties (e.g., Concord), control is important in years with ideal pathogen conditions. Fungicides with a single mode of action possess a very high potential for the development of resistance. Resistance has been observed often in the Quinone outside inhibitor (QOI) fungicides, such as strobilurins. We ascertained the levels of QoI resistance in downy mildew colonies on diseased leaves using CAPS-PCR to detect the glycine to alanine mutation (G143A) known to confer a qualitative level of resistance in fungal pathogens. Our data uncovered a small percentage of samples that contain G143A, suggesting an overall low level of QoI resistance. The low prevalence of the resistant single nucleotide polymorphism (SNP) suggests that QoI fungicides should remain a viable control mechanism in Lake Erie vineyards. Additionally, it appears that a viticultural region where tolerant hosts predominant and QoI use is minimal, resistance buildup in the pathogen population will be minimal.
\end{abstract}

\section{Introduction}

The Lake Erie Grape Belt is a 100-km by 8-km-long band along the coast of Lake Erie that contains approximately 12,000 ha of vineyards, predominately planted with Concord and Niagara grapevines. The remainder of the acreage is a mixture of Vitis vinifera and hybrid grapes used in the burgeoning winery industry. This agricultural crop provides 7000 jobs and $\$ 340$ million input into the local economy, and remains very important to the region as a whole (www.concordgrapebelt.org). The continued success and longevity of vineyard production relies on successful pathogen control.

Downy mildew [Plasmopara viticola (Berk. \& M.A. Curtis) Berl. \& De Toni] is a significant pathogen in the grape vineyard and remains a problem in climates with warm wet conditions, including in those vineyards with fairly resistant varieties, such as Concord ( $V$. hybrid 'Concord') (10). Control of this pathogen relies on a combination of host tolerance and application of pesticides, including those with quinone oxidase outside inhibitor (QoI) (FRAC code 11) activity, which includes strobilurin fungicides $(1,5)$. Strobilurins inhibit function of the cytochrome $b c 1$ complex within the electron transport chain in the mitochondria. This activity inhibits the essential energy gathering process for spore germination (1). This class of chemicals remains effective against fungal and oomycete pathogens in the vineyard, although their effectiveness is diminished by the development of resistance in pathogen populations (1). Qualitative resistance to QoI fungicides is significantly controlled by a single nucleotide polymorphism (SNP) resulting in a glycine to alanine substitution at 
position $143(\mathrm{G} 143 \mathrm{~A})$ of the cytochrome b gene (9). Previous studies have identified resistance in viticultural regions $(2,3,6,11)$, although the Lake Erie region has yet to receive a survey of G143A incidence to better understand the prevalence of resistance.

In this study, we characterized the levels of the resistant SNP (G143A) in the downy mildew population using rapid molecular techniques. The mutant allele responsible for conferring resistance was found in samples collected in the region, although the prevalence was far lower than in other viticultural regions $(2,4,7,13,16)$. Based on the detection rate in this study, QoI fungicide resistance is very low in this region, suggesting that this class of fungicides will remain an effective and valuable control method into the future as long as fungicide resistance management guidelines are followed.

\section{Sample Collection and DNA Isolation}

Samples were collected during 2009 and 2010 from production and research vineyards in Erie Co., PA, and Chautauqua Co., NY, within the Lake Erie Grape Belt (Fig. 1). All locations were managed with a conventional spray program with QoI fungicides rotated with other modes of action. Typically, if QoI fungicides were used during the growing season, it was for a single application around bloom time. Leaves with visible downy mildew lesions were collected between the months of July and October by arbitrarily sampling four leaves from the length of every third row of the selected vineyard blocks. Leaves were placed in bags with barcode labels, which were scanned using a Symbol MC55 handheld computer (Motorola Solutions Inc., Schaumburg, IL) with custom software (Code Circles LLC, East Lansing, MI) to GPS tag each sample. Leaves were stored at $-20^{\circ} \mathrm{C}$ until DNA was extracted.

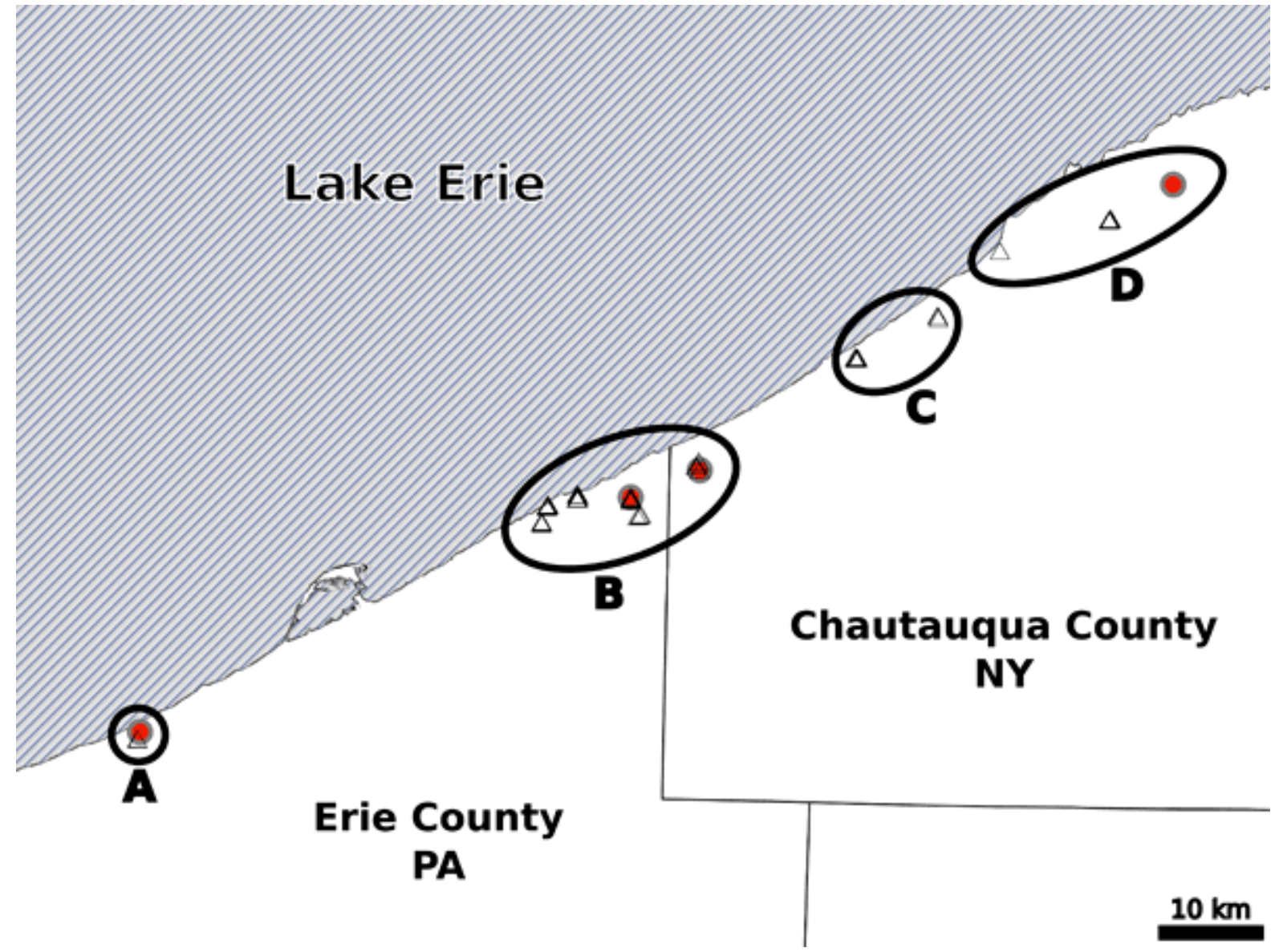

Fig. 1. Map of collections used in this project. Symbols $\Delta$ represent collections that were shown to have the wild type SNP and $\bullet$ represent each of the four samples shown to have the mutant SNP using CAPS-PCR (7). 
Mildewed leaves were ground in liquid nitrogen, and total DNA was extracted using an insect DNA isolation kit (Omega Bio-Tek Inc., Norcross, GA) following the supplied protocols. DNA concentration and quality were assessed using a BioSpec-nano spectrophotometer (Shimadzu Scientific Instruments, Columbia, MD).

\section{ARMS-Real-Time PCR}

SNP determination was carried out using Amplified Refractory Mutation System (ARMS) real-time PCR following the methods described by Baudoin et al. (2). Forward primers to detect the wild type (5' CCT TGG TGA CAA ATG AGT TTT TGG AG 3') or mutant (5' CCT TGG TGA CAA ATG AGT TTT TGG AC 3') SNPs were used in separate reactions with a common reverse primer (5' CAA CTT CTT TTC CAA TTA ATG GGA TAG 3') in a mixture including $5 \mu$ lof template, $0.5 \mathrm{mM}$ of forward and reverse primers, and $12.5 \mu$ of either $2 \times$ DyNAmo SYBR Green qPCR Mastermix (New England Biolabs, Ipswich, MA) or 2× qPCR SYBR Green Mastermix (Anaspec Inc., Freemont, CA). All samples were run on an ABI StepOne real-time system (Applied Biosystems, Carlsbad, CA) and data was analyzed with ABI StepOne v2.o software system. Samples were classified as having either the wild type SNP or the mutant SNP if the sample showed exponential amplification within the first 36 cycles of the run. Samples that were amplified with both primer sets were counted as having both SNPs present. Samples were classified to a phenotypic group once two runs gave the same result. ARMS-real-time PCR primer function was confirmed using two synthetic 82 bp oligonucleotides (wild type: CCT TGG GGA CAA ATG AGT TTT TGG GGT GCA ACA GTT ATT ACA AAT TTA TTT TCG GCT ATC CCA TTA ATT GGA AAA GAA GTT G, mutant: CCT TGG GGA CAA ATG AGT TTT TGG GㅡT GCA ACA GTT ATT ACA AAT TTA TTT TCG GCT ATC CCA TTA ATT GGA AAA GAA GTT G) (Sigma-Aldrich, St. Louis, MO) corresponding to the amplified region of the $P$. viticola cytochrome-b gene (DQ459468), which differed only at the G143A SNP. These oligomers were used at concentrations of $1 \times 10-12 \mu \mathrm{M}$, $1 \times 10-15 \mu \mathrm{M}, 1 \times 10-18 \mu \mathrm{M}$, and $1 \times 10-21 \mu \mathrm{M}$, and run with the same parameters as the ARMS-real time PCR (see above).

Samples genotyped using ARMS-real-time PCR contained 47\% (72/154) of the wild type SNP and $18 \%(28 / 154)$ of the mutant SNP. Samples that showed amplification using both primer sets (35\%) suggest that a mixed wild type and mutant population existed on that particular leaf. Samples that contain both SNPs were still considered to contribute fungicide resistant individuals to the population. When categorized for geographic location (Fig. 1), the pattern of wild type to mutant SNP distribution was fairly uniform, with each location having approximately $50 \%$ of the collected samples with the presence of the resistant SNP. Using synthetic oligomers that correspond to the ARMS-realtime PCR amplicon to test the ARMS-real-time PCR technique resulted in amplification of each template with both primers throughout the concentration range tested. Amplification of each SNP with both primers occurred even when the templates were combined at varying concentrations (data not shown).

\section{CAPS-PCR}

Cleaved Amplified Polymorphic Sequences (CAPS)-PCR was adopted from Furuya et al. (7). Each sample was amplified using the first set of nested PCR primers (forward: 5' GCC GGT ATC ATG TTA GTA GT 3', reverse: 5' GAC CTA AAG TAT TAG GGT AG $3^{\prime}$ ) with a sample mixture of $2 \mu$ lof template, $1 \mu$ of forward and reverse primer ( $0.4 \mu \mathrm{M}$ each final concentration), $8.5 \mu \mathrm{l}$ nuclease free water, and $12.5 \mu$ lof KOD $2 \times$ hotstart PCR mastermix (EMD Biosciences, San Diego, CA). Amplicons were confirmed for select samples by sequencing in the forward and reverse directions using the CAPS-PCR primers and comparing to the sequence in GenBank (data not shown). Following amplification, samples that had an amplicon of expected size were transferred to a new plate $(15 \mu \mathrm{l})$ and digested directly by adding $12 \mu \mathrm{l}$ nuclease free water, $2 \mu \mathrm{l} \mathrm{NEB}$ buffer 4 and $1 \mu \mathrm{l}$ Fnu4HI (syn. activity to Ita1) restriction enzyme (New England Biolabs, Ipswich, MA). Digests were carried out with the following program: $37^{\circ} \mathrm{C}$ for 
60 min followed by $65^{\circ} \mathrm{C}$ for $20 \mathrm{~min}$. Fragment sizes were analyzed using $1.2 \%$ agarose gels with samples stained using EZ Vision loading dye (Amresco, Solon, $\mathrm{OH}$ ) and compared to a 100 bp DNA ladder (New England Biolabs, Ipswich, MA). The digest of the initial amplicon resulted in two co-migrating products, resulting in a single shifted band if the mutant SNP was present (Fig. 2). DNA extractions from non-diseased leaves were used as a negative control, and were not amplified under the described conditions, demonstrating the specificity of the primers for downy mildew genes (Fig. 2). One sample known to have a mutant SNP was used as a positive control for all digestions. 

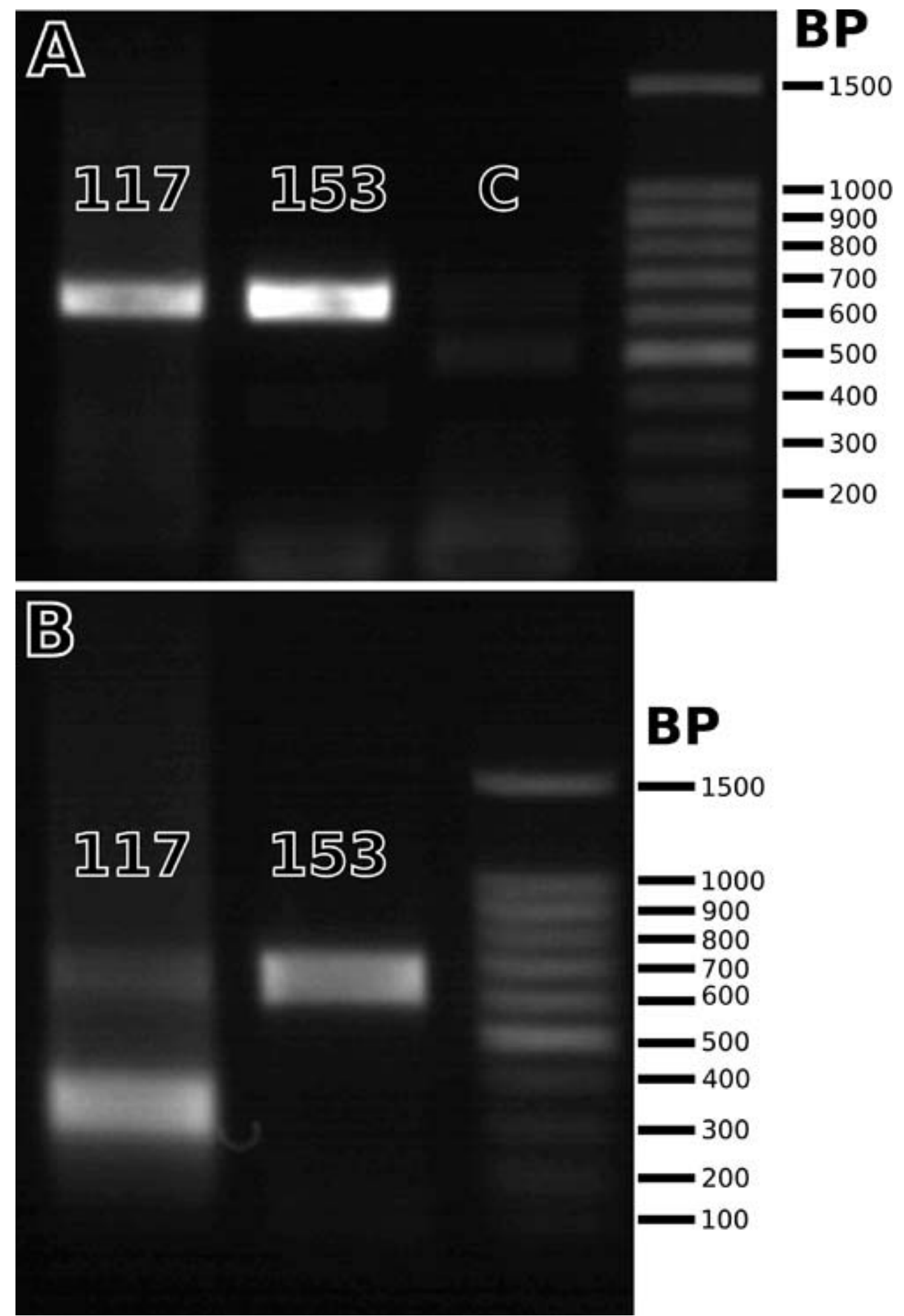

Fig. 2. Gel demonstrating the CAPS-PCR RFLP method of SNP determination. (A) Gel image of initial PCR of collected samples showing the $651 \mathrm{bp}$ band amplified from the $P$. viticola cytochrome $b$ gene. Lanes are marked with sample identification number. C denotes reaction run with DNA extracted from uninfected Vitis hybrid 'Concord' leaves demonstrating the primers do not amplify any plant genes. (B) Same samples as the above following digest with the restriction enzyme Fnu4HI (syn. Ita1). Sample 117 shows the characteristic pattern of a sample that contains the G143A SNP. The small band is a combination of the $322 \mathrm{bp}$ and $329 \mathrm{bp}$ bands that are the result of the digest. The presence of two bands is the result of a mixed wild type and mutant population on the leaf.

The CAPS-PCR method uncovered far fewer mutant SNPs than the ARMSreal-time PCR protocol among the sample population. Only 4 out of 121 samples were digested when treated with the restriction enzyme (Table 1). Also, of 
significant difference with this technique when compared with the ARMS realtime PCR is that no samples were found to contain only the mutant SNP based on the restriction pattern (Fig. 2, Table 1). There were samples that were successfully amplified with the ARMS-real-time PCR method that did not provide a product during the initial PCR amplification of the CAPS-PCR method, resulting in a smaller sample size surveyed using the latter.

Table 1. SNP distribution of samples collected from the Lake Erie Grape Belt as determined using CAPS-PCR ${ }^{\mathrm{w}}$.

\begin{tabular}{|l|c|c|c|}
\hline $\begin{array}{l}\text { Location } \\
\text { Group }^{\mathrm{x}}\end{array}$ & $\begin{array}{c}\text { Wild type } \\
(\%)^{\mathrm{Z}}\end{array}$ & $\begin{array}{c}\text { Mutant } \\
(\%)\end{array}$ & $\begin{array}{c}\text { Both } \\
(\%)\end{array}$ \\
\hline A & $3(75)$ & $0(0)$ & $1(25)$ \\
\hline B & $93(98)$ & $0(0)$ & $2(2)$ \\
\hline C & $12(100)$ & $0(0)$ & $0(0)$ \\
\hline D & $9(90)$ & $0(0)$ & $1(10)$ \\
\hline
\end{tabular}

${ }^{\mathrm{w}}$ CAPS-PCR method described in text.

$x$ Geographic groups of collections shown in Figure 1.

$y$ Number of samples for each location with specified genotype.

$\mathrm{z}$ Percent of the total number of samples collected in the location.

\section{Conclusions}

This study was undertaken with the goal of using a relatively rapid method to screen the Lake Erie viticultural region for the presence of the G143A SNP conferring qualitative resistance to QoI fungicides. While the use of activeingredient fungicides to test sensitivity of single-spore isolates likely provides the best information on which isolates are resistant, it comes at a significant cost of time and resources. The desire for a higher throughput method led to the use of published molecular methods that were designed to unambiguously determine the SNP of interest $(2,7)$.

Our attempts using the ARMS-real-time PCR method did not provide the consistent results seen in Baudoin et al. (2), possibly due to the difference in starting material (i.e., whole leaf + pathogen DNA isolations in this survey vs. pathogen spore-only DNA isolations in the previous study). Previous attempts using this ARMS-real-time PCR method on templates purified from intact mildewed leaves were also met with highly variable results (J. Colcol, personal communication). The variability observed in this work not only made interpretation of the data difficult as some samples had to be run up to five times before reaching the criteria used to apply a genotypic classification (see above), but it also significantly increased the number of real-time PCR runs required to screen the sample population, thereby multiplying the time and cost required to complete the analysis. The test using the synthetic oligomers also suggests the ARMS primer sets are not sufficient to adequately discriminate between the two SNPs, at least using our methods.

Given the variable nature of the ARMS-real-time PCR, we concluded that the data needed to be confirmed using another approach. We looked to the nestedPCR RFLP (CAPS-PCR) method published by Furuya et al. (7). This method offers an unambiguous method to discriminate between the wild type and mutant SNPs, even using whole leaf plus pathogen DNA extractions (7). We were interested in streamlining the method to allow for a faster throughput, and we were able to successfully detect SNPs based on digestion of the amplicon using the first set of the nested PCR primers, which significantly cut down on the per sample effort (7) (Fig. 2). One issue with this method was the hit-or-miss nature of the first PCR reaction, which was successful for some samples while not for others. We gave each sample three attempts at the PCR reaction before removing it from consideration and achieved moderate success on the second and third attempts. This variability is likely due to the maturity of the leaves collected from the vineyard, which were obtained in late summer or early fall, 
when PCR inhibitory compounds such as polysaccharides and polyphenolics are likely in highest concentration, especially for leaves that are infected with pathogens. The samples that were successfully amplified with PCR worked reliably for direct digestion, as demonstrated by the use of a sample known to have the mutant SNP, which was predictably cut in $100 \%$ of the digest reactions. This method allowed for the rapid throughput we were seeking, allowing us to determine the genotype of $60 \%$ of samples included in this method.

Considering these results, we conclude that the overall rate of resistance in the downy mildew population within the Lake Erie Region is lower than in previously explored viticultural regions $(2,3,6,11,16)$, although similar to rates found for some regions in Europe (14). The likely reason for this is the fairly low downy mildew susceptibility of the predominant grape variety grown in the region (i.e., Concord) and the relatively low rate of QoI fungicides used by growers (17).

Additionally, this study might provide insight into possible fitness cost of isolates that possess the G143A SNP conferring QoI resistance $(7,13,14)$. Genet et al. (8) observed that individuals who are susceptible to QoI fungicides are more fit, but also suggest that reduction or arrest of QoI use in an area with high rates of genetic-based resistance could be successful in reducing resistance in the downy mildew population over time by shifting the balance to where susceptible individuals predominate. The gains of increasing sensitivity to the fungicide can be offset by a rapid return of resistance following resumption of fungicide use (15). However, a recent study found little benefit to possessing the wild type SNP (4), possibly suggesting a naturally low level of the resistant SNP in the sampled region.

This study has shown that both QoI-resistant and -susceptible individuals can persist in vineyards receiving QoI fungicides treatments; although it appears that the low level of active ingredient use maintains an overall low level of resistant individuals. This should allow for its continued effectiveness into the future. Furthermore, the CAPS-PCR method adopted for this study allows for a rapid, cost effective way to continue to monitor for the buildup of the SNP responsible for resistance, and to ensure the continued efficacy of the QoI-class of fungicides in the Lake Erie grape region.

\section{Acknowledgments}

We gratefully thank Dr. Michael Naber for generating the map used in Figure 1. Dr. Jodi Creasap Gee and Dr. Peter Cousins are appreciatively thanked for contributing critical comments on this manuscript prior to submission. Funding was provided in part by the Penn State Erie Undergraduate Scholar Research Program.

\section{Literature Cited}

1. Bartlett, D. W., Clough, J. M., Godwin, J. R., Hall, A. A., Hamer, M., and ParrDobrzanski, B. 2002. The strobilurin fungicides. Pest Manage. Sci. 58:649-662.

2. Baudoin, A., Olaya, G., Delmotte, F., Colcol, J. F., and Sierotzki, H. 2008. QoI resistance of Plasmopara viticola and Erysiphe necator in the mid-Atlantic United States. Online. Plant Health Progress doi:10.1094/PHP-2008-0211-02-RS.

3. Chen, W.-J., Delmotte, F., Richard-Cervera, S., Douence, L., Greif, C., and CorioCostet, M.-F. 2007. At least two origins of fungicide resistance in grapevine downy mildew populations. Appl. Environ. Microbiol. 73:5162-5172.

4. Corio-Costet, M.-F., Dufour, M.-C., Cigna, J., Abadie, P., and Chen, W.-J. 2011. Diversity and fitness of Plasmopara viticola isolates resistant to QoI fungicides. Eur. J. Plant Pathol. 129:315-329.

5. Fungicide Resistance Action Committee. 2009. FRAC Code List: Fungicides sorted by mode of action. Online. FRAC, CropLife Intl., Brussels, Belgium.

6. Furuya, S., Mochizuki, M., Saito, S., Kobayashi, H., Takayanagi, T., and Suzuki, S. 2010. Monitoring of QoI fungicide resistance in Plasmopara viticola populations in Japan. Pest Manage. Sci. 66:1268-1272.

7. Furuya, S., Suzuki, S., Kobayashi, H., Saito, S., and Takayanagi, T. 2009. Rapid method for detecting resistance to a QoI fungicide in Plasmopara viticola populations. Pest Manage. Sci. 65:840-843. 
8. Genet, J.-L., Jaworska, G., and Deparis, F. 2006. Effect of dose rate and mixtures of fungicides on selection for QoI resistance in populations of Plasmopara viticola. Pest Manage. Sci. 62:188-194.

9. Gisi, U., Sierotzki, H., Cook, A., and McCaffery, A. 2002. Mechanisms influencing the evolution of resistance to Qo inhibitor fungicides. Pest Manage. Sci. 58:859867.

10. Lafon, R., and Clerjeau, M. 1988. Downy mildew. Pages 11-13 in: Compendium of Grape Diseases. R. C. Pearson and A. C. Goheeneds, eds. American Phtyopathological Society, St. Paul, MN.

11. Lodovica Gullino, M., Gilardi, G., Tinivella, F., and Garibaldi, A. 2004. Observations on the behaviour of different populations of Plasmopara viticola resistant to QoI fungicides in Italian vineyards. Phytopathol. Mediterr. 43:341-350.

12. Ma, B., and Sutton, T. B. 2007. Study on QoI sensitivity of Plasmopara viticola causing grapevine downy mildew in North Carolina. (Abstr.) Phytopathology 97:S69.

13. Matasci, C. L., Gobbin, D., Schärer, H., Tamm, L., and Gessler, C. 2008. Selection for Fungicide Resistance Throughout a Growing Season in Populations of Plasmopara viticola. Eur. J. Plant Pathol. 120:79-83.

14. Sierotzki, H., Kraus, N., Assemat, P., Stanger, C., Cleere, S., Windass, J., Gisi, U. 2005. Evolution of resistance to QoI fungicides in Plasmopara viticola populations in Europe. Page 73-80: Modern Fungicides and Antifungal Compounds IV: 14th Intl. Reinhardsbrunn Sym. British Crop Protection Council, Alton, UK.

15. Sierotzki, H., Kraus, N., Pepin, S., Ferandes, N., and Gisi, H. 2008. Dynamics of QoI resistance in Plasmopara viticola. Pages 151-157 in: Modern Fungicides and Antifungal Compounds V. H. W. Dehne, H. B. Deising, U. Gisi, K. H. Kuck, P. E. Russell, and H. Lyr, eds. DPG Selbstverlag, Braunschweig, Germany.

16. Toffolatti, S. L., Serrati, L., Sierotzki, H., Gisi, U., and Vercesi, A. 2007. Assessment of QoI resistance in Plasmopara viticola oospores. Pest Manage. Sci. 63:194-201.

17. Weigle, T. H., and Muza, A. J, eds. 2012. New York and Pennsylvania Pest Management Guidelines for Grapes. Online. PennState and Cornell Universities, Ithaca, NY. 